\title{
Decreasing Use of Chemotherapy in Older Patients With Stage III Colon Cancer Irrespective of Comorbidities
}

\author{
Viola Walter, MSc, PhDa; Daniel Boakye, MPHa; Janick Weberpals, MSc, PhDa; Lina Jansen, MSc, PhDa; \\ Walter E. Haefeli, MD'b; Uwe M. Martens, MDc; Phillip Knebel, MDd; Jenny Chang-Claude, MSc, PhDe; \\ Michael Hoffmeister, MSc, PhDa; and Hermann Brenner, MD, MPH ${ }^{a, f, g}$
}

\begin{abstract}
Background: Chemotherapy underuse in elderly patients (aged $\geq 75$ years) with colon cancer has been reported in previous studies. However, these studies were mostly registry-based and limited in their potential to consider underlying reasons of such undertreatment. This study aimed to evaluate patient and hospital determinants of chemotherapeutic treatment in patients with stage III colon cancer, with a particular focus on age and underlying reasons for nontreatment of elderly patients. Methods: A total of 629 patients with stage III colon cancer who were diagnosed in 2003 through 2012 and recruited into a populationbased study in the Rhine-Neckar region of Germany were included. Information on sociodemographic and lifestyle factors, comorbidities, and treatment was collected from patient interviews and physicians. Patient (with an emphasis on age) and hospital factors were evaluated for their associations with administration of adjuvant chemotherapy overall and of oxaliplatin specifically using multivariable logistic regression. Results: Administration of chemotherapy decreased from $94 \%$ in patients aged 30 to 64 years to $51 \%$ in those aged $\geq 75$ years. A very strong decline in chemotherapy use with age persisted even after comprehensive adjustment for multiple patient factors_-including comorbidities_-and hospital factors and was also seen among patients without any major comorbidities. Between 2005 and 2008, and 2009 and 2012, chemotherapy administration in patients aged $\geq 75$ years decreased from $60 \%$ to $41 \%$. Among chemotherapy recipients, old age was also strongly associated with higher odds of nonadministration of oxaliplatin. The 2 most commonly reported reasons for chemotherapy nonreceipt among the study population were patient refusal (30\%) and old age (24\%). Conclusions: Age was the strongest predictor of chemotherapy underuse, irrespective of comorbidities and even in patients without comorbidities. Such underuse due just to older age in otherwise healthy patients deserves increased attention in clinical practice to ensure that elderly patients also get the best possible care. Patients' refusal as the most frequent reason for chemotherapy nonreceipt also warrants further investigation to exclude misinformation as underlying cause.
\end{abstract}

J Natl Compr Canc Netw 2019;17(9):1089-1099 doi: $10.6004 /$ jnccn.2019.7287

${ }^{a}$ Division of Clinical Epidemiology and Aging Research, German Cancer Research Center, Heidelberg; ${ }^{b}$ Department of Clinical Pharmacology and Pharmacoepidemiology, University Hospital Heidelberg, Heidelberg; 'SLK-Clinics, Cancer Center Heilbronn-Franken, Heilbronn; ${ }^{\mathrm{d} D e p a r t m e n t ~ o f ~ G e n e r a l, ~ V i s c e r a l ~ a n d ~}$ Transplantation Surgery, University Hospital Heidelberg, Heidelberg; 'Division of Cancer Epidemiology, German Cancer Research Center, Heidelberg; 'Division of Preventive Oncology, German Cancer Research Center and National Center for Tumor Diseases, Heidelberg; and ${ }^{9}$ German Cancer Consortium (DKTK), German Cancer Research Center (DKFZ), Heidelberg, Germany.

\section{Background}

Colorectal cancer (CRC) incidence is particularly high in Western countries. ${ }^{1}$ In Germany, $>60,000$ people are diagnosed with CRC each year, and approximately two-thirds of the tumors are located in the colon. ${ }^{2}$ Colon cancers are primarily treated by surgery, followed by adjuvant chemotherapy under certain conditions. Adjuvant treatment of stage II colon cancer is controversial ${ }^{3}$ and mostly restricted to high-risk patients. $^{4-7}$ However, adjuvant chemotherapy has been widely recommended for patients with stage III colon cancer, beginning in the 1990s. In 2004, results from the MOSAIC trial showed that a combination of oxaliplatin with conventional 5-FU/leucovorin (LV) chemotherapy significantly improved 3-year diseasefree survival by $24 \%$ and nonsignificantly reduced 3 -year all-cause mortality by $14 \%$ compared with 5 -FU/ LV therapy alone. ${ }^{8}$ Subsequently, treatment recommendations for stage III colon cancer changed to combination therapy, also known as the FOLFOX regimen (5-FU, $\mathrm{LV}$, oxaliplatin)..$^{9,10}$

5-FU/LV alone has been shown to prolong survival throughout age groups, ${ }^{11,12}$ even in patients aged $>75$ years. ${ }^{13,14}$ However, the benefit of oxaliplatin-based therapy in elderly patients is viewed more skeptically, ${ }^{15-18}$ although Haller et al $^{19}$ suggested that the addition of oxaliplatin be considered in patients of all age groups and in those with comorbidities due to its disease-free survival benefit compared with 5-FU/LV alone. Nevertheless, registry-based studies have shown chemotherapy underuse among older patients in several countries. ${ }^{20-25}$ However, given the limited information included in cancer registries, little is known about the determinants of potential underuse of adjuvant chemotherapy.

This study evaluated the factors associated with chemotherapy administration in patients with stage III

See JNCCN.org for supplemental online content. 
colon cancer in a population-based study from Germany, in which both patient and hospital characteristics were ascertained in great detail.

\section{Methods}

\section{Study Design and Population}

This study was based on secondary analyses of data from patients with colon cancer who were diagnosed in 2003 through 2012 and recruited into the Darmkrebs: Chancen der Verhütung durch Screening (DACHS) study, an ongoing population-based case-control study performed in the southwest of Germany, with regular follow-up of participants. All patients with a first, histologically confirmed diagnosis of CRC (ICD-10 codes C18-C20) and aged $\geq 30$ years were eligible, and all had to be physically and mentally able to participate in an interview lasting for approximately 1 hour. Further details of the DACHS study have been described elsewhere. ${ }^{26-28}$ In the 22 recruiting clinics, eligible patients were informed about the study by their physicians and recruited during or shortly after their hospital stay. Data from cancer registries indicate that approximately $50 \%$ of eligible patients in the study region of approximately 2 million inhabitants were recruited. Recruitment rates were largely dependent on the clinicians in charge of recruitment in the hospitals and were slightly higher for younger versus older patients, but unlike previous studies, our study had no upper age limit. All participants provided written informed consent, and the study was approved by the ethics committees of the Medical Faculty of Heidelberg University and the state medical boards of BadenWuerttemberg and Rhineland-Palatinate.

Sociodemographic information, medical history, and lifestyle factors were obtained at baseline by trained interviewers. Details of the data collection process have been described elsewhere. ${ }^{29-32}$ Approximately 3 years after diagnosis, treatment information was obtained from general practitioner (GPs) and oncologist reports (through a standardized questionnaire and both inpatient and outpatient reports, which were extracted from the patient charts in the computer system). In case of missing information, the attending GPs/oncologists were contacted for clarification. Discharge letters were also obtained from hospitals where patients underwent colon cancer surgery, and pathology reports were obtained.

\section{Inclusion/Exclusion Criteria}

Inclusion criteria comprised a colon cancer diagnosis (ICD-10 code C18), UICC stage III disease, an R0 tumor resection, and availability of information on chemotherapy and other covariates of interest (Figure 1). Patients were included in the analysis regardless of whether

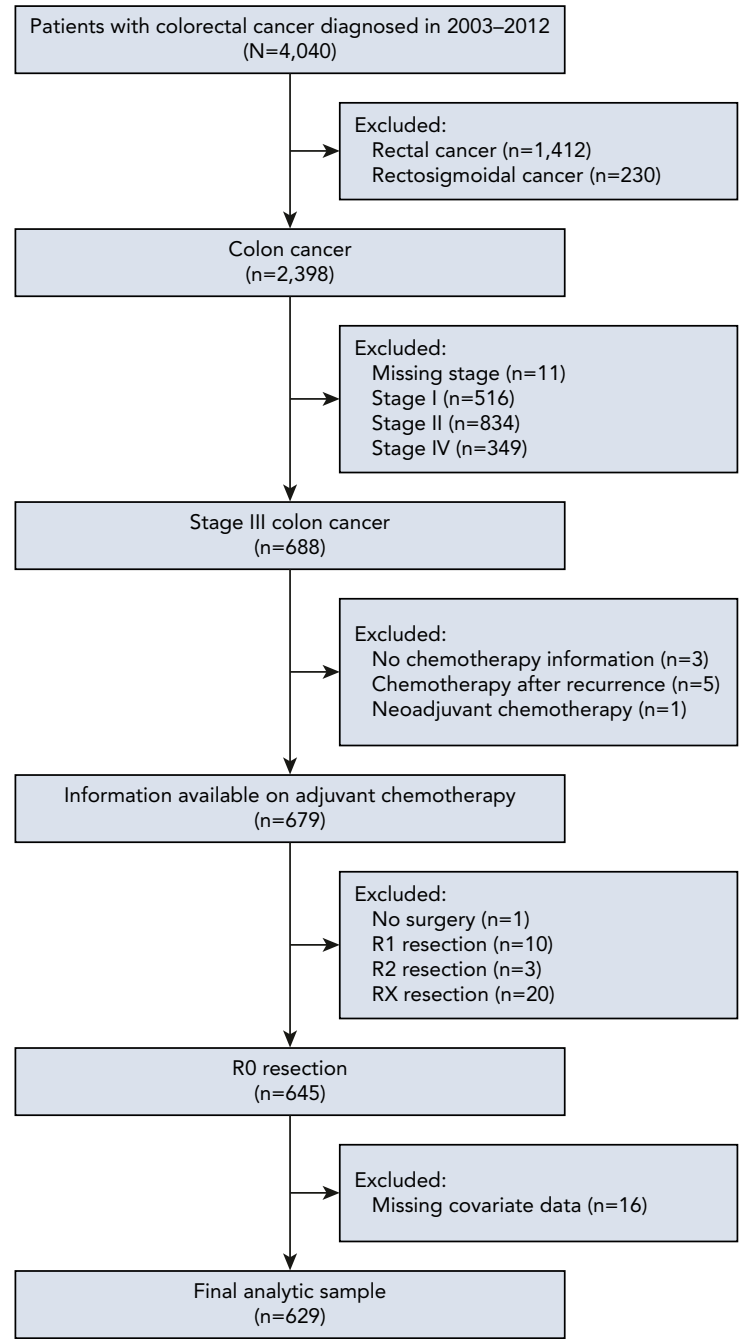

Figure 1. Flow diagram of inclusion and exclusion criteria.

they died before ascertainment of therapy information from physicians.

\section{Ascertainment of Therapeutic Information}

Information on adjuvant chemotherapy (hereafter referred to as chemotherapy) and the administered regimens and specific compounds was extracted from physician medical reports. Reasons for chemotherapy nonadministration were also extracted.

\section{Statistical Analysis}

The distribution of patient and hospital factors according to chemotherapy use was evaluated in descriptive analyses. Associations were tested for significance using chi-square tests. Factors associated with chemotherapy nonreceipt (yes/no) were investigated with multivariable logistic regression and in 2 adjustment settings: (1) adjustment for age and hospitals where patients were 


\begin{tabular}{|c|c|c|}
\hline & n (\%) & $P$ Value \\
\hline Total & $488(77.6)$ & \\
\hline Sex & & $.6325^{\mathrm{a}}$ \\
\hline Female & 234 (78.5) & \\
\hline Male & $254(76.7)$ & \\
\hline Age at diagnosis, y & & $<.0001$ \\
\hline $30-64$ & $182(94.3)$ & \\
\hline $65-74$ & $193(89.8)$ & \\
\hline$\geq 75$ & $113(51.1)$ & \\
\hline $\mathrm{BMI}^{\mathrm{b}} \mathrm{kg} / \mathrm{m}^{2}$ & & .0511 \\
\hline$<25.0$ & $189(74.1)$ & \\
\hline $25.0-29.9$ & $199(77.4)$ & \\
\hline$\geq 30.0$ & $100(85.5)$ & \\
\hline Tumor grade & & $.3664^{\circ}$ \\
\hline $1 / 2$ & $323(78.8)$ & \\
\hline $3 / 4$ & $165(75.3)$ & \\
\hline T stage & & .4398 \\
\hline $\mathrm{T} 1-2$ & $57(74.0)$ & \\
\hline T3 & $359(78.9)$ & \\
\hline T4 & $72(74.2)$ & \\
\hline $\mathrm{N}$ stage & & $.1937^{\circ}$ \\
\hline N1 & 308 (75.9) & \\
\hline N2 & $180(80.7)$ & \\
\hline Number of comorbidities ${ }^{c}$ & & $<.0001$ \\
\hline 0 & $201(86.3)$ & \\
\hline 1 & $171(78.8)$ & \\
\hline$\geq 2$ & $116(64.8)$ & \\
\hline Smoking status & & .0695 \\
\hline Never-smoker & $258(77.9)$ & \\
\hline Former smoker & $173(74.2)$ & \\
\hline Current smoker & $57(87.7)$ & \\
\hline Education level ${ }^{d}$ & & .0035 \\
\hline Low $(<9 \mathrm{y})$ & $94(67.6)$ & \\
\hline Medium (9-10 y) & $281(79.2)$ & \\
\hline $\operatorname{High}(>10 \mathrm{y})$ & $113(83.7)$ & \\
\hline Living with a partner & & $<.0001^{a}$ \\
\hline Yes & $385(81.7)$ & \\
\hline No & $103(65.2)$ & \\
\hline
\end{tabular}

Abbreviation: $\mathrm{BMI}$, body mass index; $\mathrm{CoC}$, Commission on Cancer.

(continued)

${ }^{a}$ Calculated using Fisher exact test.

b16 patients $(2.5 \%)$ were underweight (BMI $\left.<18.5 \mathrm{~kg} / \mathrm{m}^{2}\right)$.

Including diabetes, hypertension, myocardial infarction, angina pectoris,

heart failure, stroke, and cerebrovascular disorders.

dYears of school education.

eNumber of colon cancer surgeries performed per year.

\begin{tabular}{|c|c|c|}
\hline & n (\%) & $P$ Value \\
\hline Period of diagnosis & & .0037 \\
\hline 2003-2004 & $115(81.0)$ & \\
\hline 2005-2008 & $206(82.4)$ & \\
\hline 2009-2012 & $167(70.5)$ & \\
\hline Surgical volume $e^{e}$ & & .0285 \\
\hline Low $(<70)$ & $109(70.8)$ & \\
\hline Medium (70-149) & $163(82.7)$ & \\
\hline High $(\geq 150)$ & $216(77.7)$ & \\
\hline CoC-certified center status (at diagnosis/in 2017) & & .0054 \\
\hline No/No & $229(76.3)$ & \\
\hline No/Yes & $152(85.4)$ & \\
\hline Yes/Yes & 107 (70.9) & \\
\hline
\end{tabular}

Abbreviation: BMI, body mass index; CoC, Commission on Cancer.

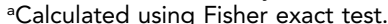

b16 patients $(2.5 \%)$ were underweight $\left(\mathrm{BMI}<18.5 \mathrm{~kg} / \mathrm{m}^{2}\right)$.

Including diabetes, hypertension, myocardial infarction, angina pectoris, heart failure, stroke, and cerebrovascular disorders.

dYears of school education.

eNumber of colon cancer surgeries performed per year.

treated, and (2) further adjustment for sex, body mass index (BMI), tumor grade, $\mathrm{T}$ stage, $\mathrm{N}$ stage, comorbidities (absolute number assessed at the time of colon cancer diagnosis, including diabetes, hypertension, myocardial infarction, angina pectoris, heart failure, stroke, and cerebrovascular disorders), smoking status, education level, living with a partner, year of diagnosis, surgical volume (number of CRC surgeries performed per year, with low defined as $<70$, medium as $70-149$, and high as $\geq 150$ ), and treatment at a Commission on Cancer (CoC)certified CRC center.

We adjusted for individual hospitals to account for potentially differing chemotherapy administration rates between hospitals. We further examined factors associated with oxaliplatin nonuse (yes/no) using multivariable logistic regression, excluding patients diagnosed in 2003 through 2004 because oxaliplatin was rarely administered during this period. In the sensitivity analysis, hierarchical logistic regression was used to account for the potential within-hospital clustering of patients and the potential between-hospital differences in chemotherapy administration.

To investigate the extent of undertreatment in elderly patients, we examined the joint effect of age and comorbidity on chemotherapy nonreceipt using multivariable logistic regression. Potential variation in the association of age with chemotherapy administration by comorbidity was examined by adding interaction terms to the model. In chemotherapy nonrecipients, reasons for chemotherapy 
nonadministration were further investigated. Data analyses were performed using SAS 9.4 (SAS Institute Inc.). Figures showing treatment patterns were created with R 3.3.2 (R Foundation for Statistical Computing). Statistical tests were 2-sided, with an alpha level of .05.

\section{Results}

Of 4,040 patients with CRC diagnosed in 2003-2012, those with rectal/rectosigmoidal cancer, stage I-II and IV colon cancer, no information regarding adjuvant chemotherapy, non-R0 resection, and missing covariate information were excluded ( $n=3,411$; Figure 1$)$.

The analytic sample comprised 629 patients, of whom $488(78 \%)$ received chemotherapy (Table 1). Administration of chemotherapy decreased with age and increasing number of comorbidities. In patients aged $<75$ years, nearly $90 \%$ received chemotherapy compared with only $51 \%$ of those aged $\geq 75$ years. In patients with $\geq 2$ comorbidities, $65 \%$ received chemotherapy compared with $>75 \%$ of those with fewer comorbidities. Moreover, chemotherapy was most frequently administered in hospitals with medium surgical volume. A summary of the hospital characteristics is shown in eTable 1 (available with this article at JNCCN.org).

\section{Chemotherapeutic Regimens and Compounds}

Administered chemotherapeutic regimens and compounds are summarized in Figure 2. FOLFOX was the most frequently administered regimen (51.4\%). Among the compounds, 5-FU (mostly in combination with LV) was the most frequently applied compound $(80 \%)$, followed by oxaliplatin (54\%). Oxaliplatin use increased significantly after 2004 . We also observed an increase in capecitabine use, which was especially administered in patients aged $\geq 75$ years, with $42 \%$ receiving it, compared with $9 \%$ of those aged $<65$ years and $18 \%$ of those aged 65 to 74 years (data not shown).

\section{Factors Associated With Chemotherapy}

Nonreceipt

In the multivariable model (Table 2), patients aged $\geq 75$ years had $>13$-fold higher odds of chemotherapy nonreceipt compared with those aged 30 to 64 years (odds ratio [OR], 13.2; 95\% CI, 6.04-28.80), even after comprehensive adjustment for multiple factors. Patients with $\geq 2$ comorbidities had significantly higher odds of chemotherapy nonreceipt than those with no comorbidities (OR, 2.55; 95\% CI, 1.36-4.78).

Compared with patients with BMI $<25 \mathrm{~kg} / \mathrm{m}^{2}$, chemotherapy nonreceipt was much less common in those with $\mathrm{BMI} \geq 30.0 \mathrm{~kg} / \mathrm{m}^{2}$ (OR, 0.48; 95\% CI, 0.23-0.98). Patients not living with a partner and those diagnosed in 2009-2012 had approximately $80 \%$ and $110 \%$ higher odds of not receiving chemotherapy, respectively. Patients treated in medium-volume hospitals had lower odds of chemotherapy nonreceipt compared with low-volume hospitals (OR, 0.44; 95\% CI, 0.24-0.82). Patients with a higher educational level and those who received treatment in hospitals that received $\mathrm{CoC}$ certification for CRC care after patients' diagnosis had nonsignificant lower odds of chemotherapy nonreceipt.

\section{Factors Associated With Oxaliplatin Nonuse}

Table 3 shows the determinants of oxaliplatin nonuse in chemotherapy recipients. Old age was strongly associated with oxaliplatin nonuse, with adjusted ORs of 7.00 (95\% CI, 2.87-17.1) for patients aged 65 to 74 years and 80.4 (95\% CI, 28.3-228.6) for those aged $\geq 75$ years
A

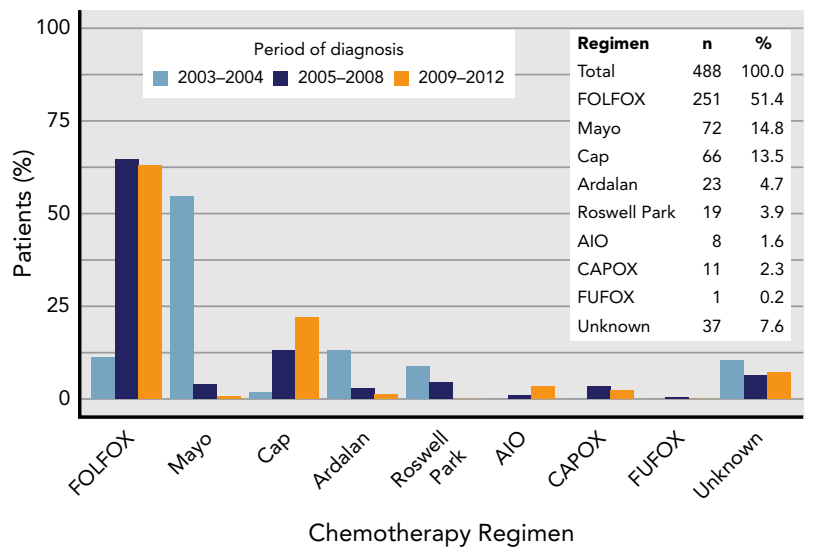

B

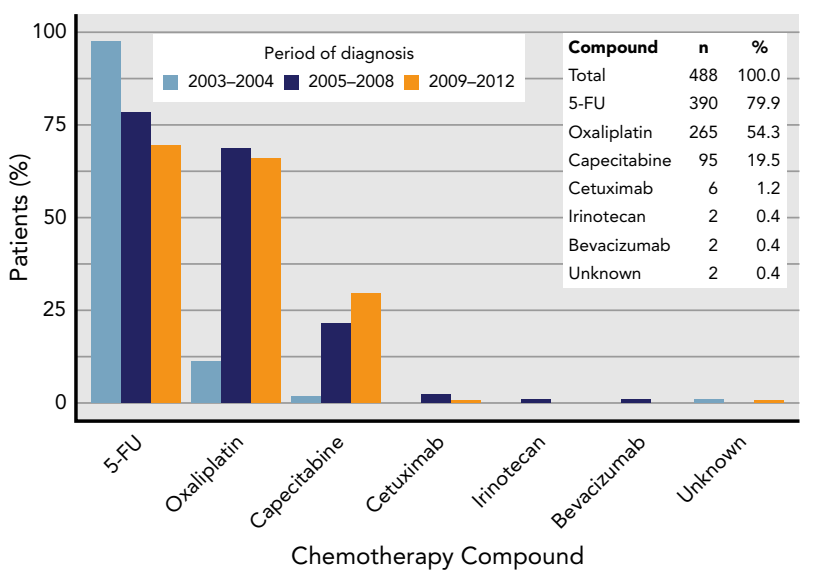

Figure 2. Administered chemotherapy (A) regimens and (B) compounds in patients with stage III colon cancer, overall and by period of diagnosis. Abbreviations: AIO, 5-FU/leucovorin; Ardalan, 5-FU/leucovorin; Cap, capecitabine monotherapy; CAPOX, capecitabine/oxaliplatin; FOLFOX, 5-FU/leucovorin/ oxaliplatin; FUFOX, 5-FU/leucovorin/oxaliplatin; Mayo, 5-FU/leucovorin; Roswell Park, 5-FU/leucovorin. 
Table 2. Factors Associated With Nonadministration of Adjuvant Chemotherapy

No Chemotherapy vs Chemotherapy

\begin{tabular}{|c|c|c|c|c|}
\hline & \multirow[b]{2}{*}{$\begin{array}{c}\text { Patients } \\
\text { n }\end{array}$} & \multirow[b]{2}{*}{$\begin{array}{c}\text { Events } \\
n\end{array}$} & \\
\hline & & & $\mathrm{OR}^{\mathrm{a}}(95 \% \mathrm{Cl})$ & $\mathrm{OR}^{\mathrm{b}}(95 \% \mathrm{Cl})$ \\
\hline \multicolumn{5}{|l|}{ Sex } \\
\hline Female & 298 & 64 & Ref & Ref \\
\hline Male & 331 & 77 & $1.26(0.81-1.97)$ & $1.59(0.86-2.94)$ \\
\hline \multicolumn{5}{|l|}{ Age at diagnosis, y } \\
\hline $30-64$ & 193 & 11 & Ref & Ref \\
\hline $65-74$ & 215 & 22 & $1.98(0.92-4.27)$ & $1.67(0.73-3.83)$ \\
\hline$\geq 75$ & 221 & 108 & $16.7(8.35-33.3)$ & $13.2(6.04-28.80)$ \\
\hline \multicolumn{5}{|l|}{$\mathrm{BMI}, \mathrm{kg} / \mathrm{m}^{2}$} \\
\hline$<25.0$ & 255 & 66 & Ref & Ref \\
\hline $25.0-29.9$ & 257 & 58 & $0.77(0.47-1.24)$ & $0.75(0.44-1.28)$ \\
\hline$\geq 30.0$ & 117 & 17 & $0.57(0.29-1.11)$ & $0.48(0.23-0.98)$ \\
\hline \multicolumn{5}{|l|}{ Tumor grade } \\
\hline $1 / 2$ & 410 & 87 & Ref & Ref \\
\hline $3 / 4$ & 219 & 54 & $1.17(0.74-1.85)$ & $1.31(0.79-2.16)$ \\
\hline \multicolumn{5}{|l|}{ T stage } \\
\hline T1-2 & 77 & 20 & Ref & Ref \\
\hline T3 & 455 & 96 & $0.73(0.38-1.43)$ & $0.72(0.35-1.47)$ \\
\hline T4 & 97 & 25 & $1.02(0.44-2.35)$ & $1.02(0.40-2.58)$ \\
\hline \multicolumn{5}{|l|}{$\mathrm{N}$ stage } \\
\hline N1 & 406 & 98 & Ref & Ref \\
\hline N2 & 223 & 43 & $0.66(0.41-1.07)$ & $0.65(0.39-1.09)$ \\
\hline \multicolumn{5}{|c|}{ Number of comorbidities ${ }^{c}$} \\
\hline 0 & 233 & 32 & Ref & Ref \\
\hline 1 & 217 & 46 & $1.22(0.68-2.20)$ & $1.18(0.64-2.19)$ \\
\hline$\geq 2$ & 179 & 63 & $2.37(1.32-4.26)$ & $2.55(1.36-4.78)$ \\
\hline \multicolumn{5}{|l|}{ Smoking status } \\
\hline Never-smoker & 331 & 73 & Ref & Ref \\
\hline Former smoker & 233 & 60 & $1.60(1.00-2.56)$ & $1.54(0.88-2.70)$ \\
\hline Current smoker & 65 & 8 & $1.24(0.49-3.13)$ & $1.04(0.39-2.77)$ \\
\hline \multicolumn{5}{|l|}{ Education level ${ }^{d}$} \\
\hline Low $(<9$ y) & 139 & 45 & Ref & Ref \\
\hline Medium (9-10 y) & 355 & 74 & $0.97(0.58-1.64)$ & $0.95(0.53-1.71)$ \\
\hline High (>10 y) & 135 & 22 & $0.58(0.29-1.13)$ & $0.47(0.21-1.04)$ \\
\hline \multicolumn{5}{|l|}{ Living with a partner } \\
\hline Yes & 471 & 86 & Ref & Ref \\
\hline No & 158 & 55 & $1.50(0.92-2.42)$ & $1.77(1.00-3.12)$ \\
\hline
\end{tabular}

Abbreviations: BMI, body mass index; CoC, Commission on Cancer; OR, odds ratio.

(continued on next page)

aAdjusted for age and hospital where patients were treated.

${ }^{b}$ Additional adjustment for sex, BMI, T stage, N stage, number of comorbidities, smoking status, education level, living with a partner, and period of diagnosis.

Including diabetes, hypertension, myocardial infarction, angina pectoris, heart failure, stroke, and cerebrovascular disorders.

dYears of school education.

eHospital where patients received treatment was removed from the model. 
Table 2. Factors Associated With Nonadministration of Adjuvant Chemotherapy (cont.)

\begin{tabular}{|c|c|c|c|c|}
\hline & \multirow[b]{2}{*}{$\begin{array}{c}\text { Patients } \\
n\end{array}$} & \multirow[b]{2}{*}{$\begin{array}{c}\text { Events } \\
\text { n }\end{array}$} & \multicolumn{2}{|c|}{ No Chemotherapy vs Chemotherapy } \\
\hline & & & $O R^{a}(95 \% \mathrm{Cl})$ & $\mathrm{OR}^{\mathrm{b}}(95 \% \mathrm{Cl})$ \\
\hline 2003-2004 & 142 & 27 & Ref & Ref \\
\hline 2005-2008 & 250 & 44 & $0.96(0.51-1.80)$ & $0.86(0.44-1.67)$ \\
\hline Low & 154 & 45 & Ref & Ref \\
\hline Medium & 197 & 34 & $0.56(0.31-0.98)$ & $0.44(0.24-0.82)$ \\
\hline High & 278 & 62 & $0.75(0.45-1.25)$ & $0.67(0.39-1.14)$ \\
\hline \multicolumn{5}{|c|}{ CoC-certified center status (at diagnosis/in 2017) } \\
\hline
\end{tabular}

Abbreviations: BMI, body mass index; CoC, Commission on Cancer; OR, odds ratio.

${ }^{a}$ Adjusted for age and hospital where patients were treated.

${ }^{b}$ Additional adjustment for sex, BMI, T stage, N stage, number of comorbidities, smoking status, education level, living with a partner, and period of diagnosis. Including diabetes, hypertension, myocardial infarction, angina pectoris, heart failure, stroke, and cerebrovascular disorders.

dYears of school education.

eHospital where patients received treatment was removed from the model.

compared with younger patients. Patients without a partner had 2.3-fold increased odds of oxaliplatin nonuse, and those treated in medium-volume versus lowvolume hospitals and hospitals that received $\mathrm{CoC}$ certification for CRC care after patients' diagnosis versus no certification had $56 \%$ and $58 \%$ lower odds of oxaliplatin nonuse, respectively. Results from hierarchical logistic models for the determinants of chemotherapy nonreceipt and oxaliplatin nonuse were comparable to those from logistic regression models.

\section{Joint Effect of Age and Comorbidity on \\ Chemotherapy Administration}

Table 4 shows the frequency of chemotherapy use according to age and comorbidity. Patients aged $\geq 75$ years received chemotherapy much less often than younger patients, even when none of the assessed comorbidities were present. Multivariable logistic regression analysis of the joint effect of age and comorbidity on chemotherapy nonreceipt showed similar results (Table 5). Among patients aged $<75$ years, comorbidities were not associated with chemotherapy nonreceipt, whereas age $\geq 75$ was strongly associated with chemotherapy nonreceipt even in the absence of comorbidities, but the interaction test showed no significant result $(P=.5452)$.

\section{Trend of Chemotherapy Use}

Chemotherapy administration decreased over time from $81 \%$ in 2003-2004 to $71 \%$ in 2009-2012 ( $P_{\text {Cochran-Armitage trend }}=.0069$;
Table 6). In patients aged $<75$ years, the decrease was more moderate $\left(P_{\text {Cochran-Armitage trend }}=.1885\right)$, whereas in those aged $\geq 75$ years, administration decreased from $60 \%$ in 2005-2008 to $41 \%$ in 2009-2012 ( $\left.P_{\text {Cochran-Armitage trend }}=.0149\right)$.

\section{Reasons for Chemotherapy Nonreceipt}

Reasons for not administering chemotherapy to some patients, as reported by physicians, are shown in eTable 2 . The 3 most commonly reported reasons were patient refusal $(30 \%)$, old age $(24 \%)$, and poor health (17\%). No strong associations were observed between patient and hospital characteristics and patient refusal, but refusal seemed to be more common in recent versus earlier years (eTable 3). Sensitivity analysis of factors associated with chemotherapy nonadministration excluded patients who refused chemotherapy $(n=42)$. The associations for old age, comorbidity, and year of diagnosis were slightly attenuated but remained highly significant, whereas those for living with a partner and treatment in a medium-volume hospital became stronger (eTable 4). The association for higher education level also became stronger and reached statistical significance.

\section{Discussion}

In this multicenter cohort of patients from Germany with stage III colon cancer, older age was strongly associated with chemotherapy nonreceipt, irrespective of comorbidities and other potential determinants. 


\section{Table 3. Associations of Patient and Hospital Factors With Nonadministration of Oxaliplatin}

Total $(\mathbf{N}=372)^{a}$

\section{Variable}

Sex

\section{Female}

Male

Age at diagnosis, $y$

\begin{tabular}{lr}
\hline $30-64$ & 136 \\
\hline $65-74$ & 149 \\
$\geq 75$ & 87
\end{tabular}

BMI, $\mathrm{kg} / \mathrm{m}^{2}$

$<25.0$
$25.0-29.9$
$\geq 30.0$

$\mathbf{N}$

136

149

87

153
153
66

$\begin{array}{ll}173 & 57(33.0) \\ 199 & 63(31.7)\end{array}$

252

120

43

271

14 (32.6)

T1-2

T3

T4

$\mathrm{N}$ stage

$\mathrm{N} 1$

N2

Number of comorbidities ${ }^{d}$

0
1
$\geq 2$

Smoking status

Never-smoker
Former smoker
Current smoker

Education levele

Low $(<9$ y)
Medium (9-10 y)
High $(>10$ y)

Living with a partner

Yes

Period of diagnosis

\begin{tabular}{lll}
\hline $2005-2008$ & 206 & $64(31.0)$ \\
\hline $2009-2012$ & 166 & $56(33.7)$
\end{tabular}

\begin{tabular}{rl}
292 & $84(28.8)$ \\
80 & $36(45.0)$ \\
\hline 206 & $64(31.0)$ \\
166 & $56(33.7)$ \\
\hline
\end{tabular}

$\begin{array}{rl}69 & 33(47.8) \\ 210 & 63(30.0) \\ 93 & 24(25.8)\end{array}$

$41(47.7)$

66 (37.1)

$45(31.0)$

9 (18.4)

No Oxaliplatin vs Oxaliplatin $\mathrm{OR}^{\mathrm{b}}(95 \% \mathrm{Cl})$ $\operatorname{OR}^{\mathrm{c}}(95 \% \mathrm{Cl})$

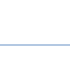

$\begin{array}{cc}\text { Ref } & \text { Ref } \\ 0.94(0.52-1.70) & 1.20(0.57-2.54)\end{array}$

Ref

\begin{tabular}{ll}
$7.05(3.14-15.8)$ & $7.00(2.87-17.1)$ \\
\hline $81.3(30.8-214.3)$ & $80.4(28.3-228.6)$
\end{tabular}

Ref

Ref

$1.14(0.59-2.20) \quad 1.11(0.55-2.26)$

$2.17(0.93-5.09)$

$2.21(0.88-5.55)$

$0.9)$

(2)

$0.86(0.45-1.61) \quad 0.80(0.40-1.60)$

.8)

Ref

$\begin{array}{ll}1.21(0.49-3.01) & 1.30(0.47-3.58) \\ 1.20(0.39-3.74) & 2.01(0.55-7.32)\end{array}$

$1.20(0.39-3.74) \quad 2.01(0.55-7.32)$

Ref

Ref

$0.71(0.38-1.32) \quad 0.64(0.33-1.25)$

\begin{tabular}{rrrc}
150 & $37(24.7)$ & Ref & Ref \\
136 & $42(30.9)$ & $0.75(0.37-1.51)$ & $0.63(0.30-1.34)$ \\
\hline 86 & $41(47.7)$ & $1.56(0.73-3.37)$ & $1.38(0.60-3.20)$ \\
\hline 178 & & & Ref \\
145 & $66(37.1)$ & $0.98(0.52-1.84)$ & $1.07(0.51-2.22)$ \\
\hline 49 & $45(31.0)$ & $0.81(0.30-2.20)$ & $0.74(0.25-2.19)$ \\
\hline
\end{tabular}

Abbreviations: BMI, body mass index; $\mathrm{CoC}$, Commission on Cancer; OR, odds ratio.

apatients diagnosed in 2003-2004 were excluded ( $n=116)$.

${ }^{b}$ Adjusted for age and treating hospital.

'Additional adjustment for sex, BMI, T stage, $\mathrm{N}$ stage, number of comorbidities, smoking status, education level, living with a partner, and period of diagnosis.

Including diabetes, hypertension, myocardial infarction, angina pectoris, heart failure, stroke, and cerebrovascular disorder.

eYears of school education.

fHospital where patients received treatment was removed from the model. 


\begin{tabular}{|c|c|c|c|c|}
\hline \multirow[b]{2}{*}{ Variable } & \multirow{2}{*}{$\frac{\text { Total }(\mathbf{N}=\mathbf{3 7 2})^{\mathrm{a}}}{\mathbf{N}}$} & \multirow{2}{*}{$\frac{\text { Did Not Receive Oxaliplatin }(n=120)}{n(\%)}$} & \multicolumn{2}{|c|}{ No Oxaliplatin vs Oxaliplatin } \\
\hline & & & $\mathrm{OR}^{\mathrm{b}}(95 \% \mathrm{CI})$ & ORc $(95 \% \mathrm{Cl})$ \\
\hline \multicolumn{5}{|c|}{ Surgical volume ${ }^{f}$} \\
\hline Low & 75 & $33(44.0)$ & Ref & Ref \\
\hline Medium & 124 & $32(25.8)$ & $0.51(0.25-1.06)$ & $0.44(0.20-0.96)$ \\
\hline High & 173 & $55(31.8)$ & $0.63(0.32-1.25)$ & $0.60(0.29-1.22)$ \\
\hline \multicolumn{5}{|c|}{ CoC-certified center status (at diagnosis/in 2017) } \\
\hline No/No & 163 & $55(33.7)$ & Ref & Ref \\
\hline No/Yes & 103 & $23(22.3)$ & $0.44(0.22-0.88)$ & $0.42(0.20-0.87)$ \\
\hline Yes/Yes & 106 & $42(39.6)$ & $1.28(0.68-2.39)$ & $1.16(0.59-2.32)$ \\
\hline
\end{tabular}

Abbreviations: BMI, body mass index; CoC, Commission on Cancer; OR, odds ratio.

aPatients diagnosed in 2003-2004 were excluded $(n=116)$.

${ }^{\mathrm{b}}$ Adjusted for age and treating hospital.

'Additional adjustment for sex, BMI, T stage, N stage, number of comorbidities, smoking status, education level, living with a partner, and period of diagnosis.

Including diabetes, hypertension, myocardial infarction, angina pectoris, heart failure, stroke, and cerebrovascular disorder.

eYears of school education.

${ }^{f}$ Hospital where patients received treatment was removed from the model.

In particular, age $\geq 75$ years was strongly associated with chemotherapy nonreceipt, even when no major comorbidity was present, and chemotherapy administration in this age group furthermore decreased over time.

Potential underuse of chemotherapy in elderly patients with colon cancer has been reported in previous studies. ${ }^{20-25,33}$ However, these studies were mostly registrybased $^{20-24,33}$ and therefore largely limited in terms of other potentially influential factors associated with chemotherapy use that they could have considered. This population-based study aimed to provide important and comprehensive insight into possible patient- and hospital-based determinants of chemotherapy administration. It also examined chemotherapeutic undertreatment in elderly patients in detail and, as one of few studies, reported potential underlying reasons for nontreatment ${ }^{34}$ and further assessed the joint effect of age and comorbidity. ${ }^{35}$

A review of US patients with stage III colon cancer diagnosed in 1991 through 2001 found that older age, female sex, diagnosis in recent years, being unmarried, and presence of comorbidities were associated with lower likelihood of receiving chemotherapy. ${ }^{21}$ Looking at patient factors, our analysis showed similar results, but further broadened the investigative spectrum to healthcare settings outside the United States and to a more recent time period. The associations for age indicate a potential undertreatment in older patients, despite the reported survival benefits from chemotherapy. ${ }^{11-14,24}$ We found that the frequency of chemotherapy administration was significantly lower in patients with $\geq 2$ comorbidities, which agrees with findings from previous studies. ${ }^{20,21,23,25}$
However, strong associations between age and chemotherapy nonreceipt persisted even after comprehensive adjustment for this important covariate. Chemotherapy use at older age was even substantially lower in recent years than in earlier time periods. The reasons for this trend are unclear and warrant further investigation.

Previous studies rarely investigated reasons for and the joint effect of age and comorbidity on chemotherapy nonreceipt. We observed that even in the absence of comorbidities, patients aged $\geq 75$ years were much less likely to receive chemotherapy than younger patients; for instance, of 18 patients in this category who did not receive chemotherapy, 6 were explicitly reported not to have received chemotherapy due to old age. Nevertheless, frailty, poor functional status, or contraindications to chemotherapy may have influenced whether a patient received chemotherapy, irrespective of the comorbidities evaluated in our study. In a previous study, Ko et $\mathrm{al}^{34}$ reported comorbidities $(60 \%)$, old age $(45 \%)$, and patient choice $(32 \%)$ as the most frequent reasons for chemotherapy nonreceipt in patients aged $\geq 70$ years, whereas the most commonly reported reasons in our study were patient choice $(30 \%)$, old age $(24 \%)$, and poor health $(17 \%)$. However, the association for old age and chemotherapy nonreceipt was only attenuated slightly when patients who refused chemotherapy were excluded from the analysis.

Reasons for refusal of treatment are unknown, but patients are likely to be influenced by recommendations from their oncologist. ${ }^{36}$ Our analysis also showed higher refusal rates in patients diagnosed in more recent 


\begin{tabular}{|c|c|c|c|}
\hline \multirow[b]{2}{*}{ Age at Diagnosis } & \multicolumn{3}{|c|}{ Number of Comorbid Conditions ${ }^{a}$} \\
\hline & $\%$ (n Treated/n Total) & $\%$ (n Treated/n Total) & $\%$ (n Treated/n Total) \\
\hline$\geq 75 y$ & $64.0 \%(32 / 50)$ & $55.6 \%(45 / 81)$ & $40.0 \%(36 / 90)$ \\
\hline
\end{tabular}

alncluding diabetes, hypertension, myocardial infarction, angina pectoris, heart failure, stroke, and cerebrovascular disorders.

years. Patient choice as one of the most important influential factors for not receiving chemotherapy should be investigated more closely to exclude misinformation as an underlying cause. The potential undertreatment of elderly patients without comorbidities should also be investigated more closely.

Regarding hospital factors, previous studies have investigated volume and type, ${ }^{22,33,37,38}$ but have reported heterogeneous results. No significant associations have been reported for hospital volume, but lower odds of chemotherapy administration in patients treated at teaching hospitals have been previously noted..$^{22,37}$ Daly et $\mathrm{a}^{33}$ found that low surgical volume was associated with undertreatment of patients with stage III colon cancer, and that these patients were more likely to receive chemotherapy in academic/research institutions. In another study, ${ }^{38}$ patients with stage III colon cancer treated in hospitals with a CoC-certified cancer program were more likely to receive chemotherapy. Our analysis found treatment at medium-volume hospitals to be significantly associated with higher odds of chemotherapy administration.

Oxaliplatin is administered in most patients with stage III colon cancer. Haller et al $^{19}$ even suggested consideration of oxaliplatin in all age groups, considering its disease-free survival benefit compared with 5-FU/LV therapy alone. No previous study has investigated the determinants of oxaliplatin administration, although advanced age was the strongest predictor of its nonuse in our analysis. In addition, patients living without a partner and those treated in low-volume hospitals and in hospitals without a certified CRC program had higher odds of oxaliplatin nonuse.

Reasons for the trend in decreasing chemotherapy use overall and in older adults are unclear. However, one explanation could be the observed increase in patient refusal rates. Additionally, the frequency of comorbidities seems to be increasing, ${ }^{39}$ as observed in our study, possibly due to enhanced comorbidity assessments. Finally, due to the small amount of evidence showing the benefits of oxaliplatin-based therapy in older patients ${ }^{40}$ coupled with the potentially high risk/ benefit ratio of chemotherapy in older patients, it is possible that eligibility criteria for chemotherapy have become more stringent in recent years.

Major strengths of our study include its use of a population-based sample and inclusion of a thorough ascertainment of therapy data. We evaluated and

Table 5. Joint Association of Age and Comorbidity With Odds of Not Receiving Adjuvant Chemotherapy

\begin{tabular}{|c|c|c|c|c|}
\hline \multirow[b]{2}{*}{ Age at Diagnosis + Comorbidities ${ }^{a}$} & \multirow[b]{2}{*}{ Patients, $\mathbf{n}$} & \multirow[b]{2}{*}{ Events, $\mathbf{n}$} & \multicolumn{2}{|c|}{ No Chemotherapy vs Chemotherapy } \\
\hline & & & $\mathrm{OR}^{\mathrm{b}}(95 \% \mathrm{Cl})$ & $\mathrm{OR}^{\mathrm{c}}(95 \% \mathrm{Cl})$ \\
\hline$<75 y+0$ comorbidities & 183 & 14 & Ref & Ref \\
\hline$<75 y+1$ comorbidity & 136 & 10 & $0.93(0.40-2.18)$ & $0.94(0.38-2.32)$ \\
\hline$<75 y+>1$ comorbidity & 89 & 9 & $1.47(0.60-3.58)$ & $1.84(0.71-4.75)$ \\
\hline$\geq 75$ y +0 comorbidities & 50 & 18 & $5.89(2.59-13.35)$ & $6.54(2.73-16.65)$ \\
\hline$\geq 75 y+1$ comorbidity & 81 & 36 & $10.11(4.91-20.83)$ & $10.14(4.64-22.17)$ \\
\hline$\geq 75 y+>1$ comorbidity & 90 & 54 & $22.79(10.90-47.62)$ & $23.23(10.34-52.23)$ \\
\hline & & & $P_{\text {interaction }}=.2602^{\mathrm{d}}$ & $P_{\text {interaction }}=.5452^{\mathrm{d}}$ \\
\hline
\end{tabular}

ancluding diabetes, hypertension, myocardial infarction, angina pectoris, heart failure, stroke, and cerebrovascular disorders.

${ }^{b}$ Adjusted for hospitals where patients were treated.

cAdditional adjustment for body mass index, tumor grade, T stage, $\mathrm{N}$ stage, smoking status, education status, living with a partner, and period of diagnosis.

${ }^{\mathrm{d}} P_{\text {interaction }}$ assesses the multiplicative effect of age and comorbidity on chemotherapy administration. 
Table 6. Probability of Receiving Chemotherapy

\begin{tabular}{|c|c|c|c|}
\hline Period of Diagnosis & Overall (Proportion) & \multicolumn{2}{|c|}{ Age at Diagnosis } \\
\hline $2003-2004$ & $81.0 \%(115 / 142)$ & $93.7 \%(89 / 95)$ & $55.3 \%(26 / 47)$ \\
\hline $2005-2008$ & $82.4 \%(206 / 250)$ & $93.4 \%(156 / 167)$ & $60.2 \%(50 / 83)$ \\
\hline 2009-2012 & $70.5 \%(167 / 237)$ & $89.0 \%(130 / 146)$ & $40.7 \%(37 / 91)$ \\
\hline
\end{tabular}

adjusted for several clinically relevant variables related to chemotherapy administration, including factors not previously covered. Data collection by trained interviewers and a thorough follow-up led to a very low proportion of missing values in these data. The clustering of patients within hospitals was adequately addressed by adjusting for individual hospitals where patients were treated. We also explored the determinants of oxaliplatinbased therapy and assessed physician reasons for not administering chemotherapy to some patients in order to identify potential starting points for increasing rates of chemotherapy acceptance and use.

However, our study also had limitations. We were unable to consider dosage or duration of chemotherapy in these analyses. Because most of the hospitals included were teaching hospitals, the study sample may have received a higher standard of treatment than patients in other studies. Despite comprehensive adjustment for potential confounders, we cannot exclude the possibility of residual confounding by factors potentially associated with chemotherapy administration but not adjusted for, such as functional status, microsatellite instability, enrollment in clinical trials, or postoperative complications. In particular, contraindications to chemotherapy, such as reduced renal clearance, are more common at older age. However, it seems unlikely that residual confounding by such factors could explain the strong association between old age and chemotherapy underuse persisting even in the fully adjusted models. Finally, although comorbidity seemed to have a substantial impact on chemotherapy administration in older but not younger patients, the interaction tests showed no significant results. This may be because of the small sample size or the assessment of interaction on a multiplicative scale only.

\section{Conclusions}

In this population-based sample of patients with stage III colon cancer in the Rhine-Neckar region of Germany, age was the strongest predictor of chemotherapy nonadministration, irrespective of comorbidity and other potential determinants. In particular, old age was a strong predictor of chemotherapy undertreatment, even in patients with no major comorbidities. This finding corroborates the still apparent issue of undertreatment in healthy older patients, although several studies have shown that the elderly could benefit well from chemotherapy. Further investigation is required to identify the underlying reasons for nonadherence to recommended adjuvant therapy in elderly patients whose health and preferences would allow for treatment.

\section{Acknowledgments}

The authors wish to thank Ute Handte-Daub, Ansgar Brandhorst, and Petra Bächer for their excellent technical assistance. The authors express their appreciation to the study participants and the interviewers who collected the data. The authors also thank the following hospitals and cooperating institutions that recruited patients for this study: Chirurgische Universitätsklinik Heidelberg, Klinik am Gesundbrunnen Heilbronn, St. Vincentiuskrankenhaus Speyer, St. Josefskrankenhaus Heidelberg, Chirurgische Universitätsklinik Mannheim, Diakonissenkrankenhaus Speyer, Krankenhaus Salem Heidelberg, Kreiskrankenhaus Schwetzingen, St. Marienkrankenhaus Ludwigshafen, Klinikum Ludwigshafen, Stadtklinik Frankenthal, Diakoniekrankenhaus Mannheim, Kreiskrankenhaus Sinsheim, Klinikum am Plattenwald Bad Friedrichshall, Kreiskrankenhaus Weinheim, Kreiskrankenhaus Eberbach, Kreiskrankenhaus Buchen, Kreiskrankenhaus Mosbach, Enddarmzentrum Mannheim, Kreiskrankenhaus Brackenheim, and the Cancer Registry of Rhineland-Palatinate, Mainz.

Submitted August 3, 2018; accepted for publication February 21, 2019.

Author contributions: Study design: Walter, Jansen, Chang-Claude, Hoffmeister, Brenner. Data analysis and interpretation: Walter, Boakye, Weberpals, Jansen, Hoffmeister, Brenner. Contributed new analytical methods: Walter, Boakye, Weberpals, Jansen, Haefeli, Martens, Knebel, Chang-Claude. Manuscript preparation: Walter, Boakye, Jansen, Brenner. Manuscript review and approval: All authors.

Disclosures: Dr. Haefeli has disclosed that he owns equity interest/stock options in Dosing $\mathrm{GmbH}$, his spouse is an employee of Dosing $\mathrm{GmbH}$, and he has received grant/research support from ADIR, Bayer AG, Boehring, GlaxoSmithKline, Hepatera Ltd, Janssen GmbH, MYR Pharmaceuticals, and QPS. The remaining authors have disclosed that they have not received any financial consideration from any person or organization to support the preparation, analysis, results, or discussion of this article.

Funding: This work was supported by grants from the German Research Council (BR 1704/6-1, BR 1704/6-3, BR 1704/6-4, CH 117/1-1) and the German Federal Ministry of Education and Research (01KH0404, 01ER0814, 01ER0815, 01ER1505A, 01ER1505B)

Correspondence: Lina Jansen, MSc, PhD, Division of Clinical Epidemiology and Aging Research, German Cancer Research Center, Im Neuenheimer Feld 581, Heidelberg, 69120, Germany. Email: I.jansen@dkfz-heidelberg.de 


\section{References}

1. Ferlay J, Soerjomataram I, Ervik M. GLOBOCAN 2012 v1.0, Cancer Incidence and Mortality Worldwide: IARC CancerBase No. 11 [Internet]. Lyon, France: International Agency for Research on Cancer; 2013. Accessed February 28, 2018.

2. Kaatsch P, Spix C, Katalinic A, et al. Krebs in Deutschland 2011/2012. Berlin, Germany: Robert Koch-Institut; 2015.

3. McGee MF, Benson AB III. Adjuvant chemotherapy for stage II colon cancer: everyone still needs a tailor. Ann Surg Oncol 2014;21:1765-1767.

4. Chang GJ, Kaiser AM, Mills S, et al. Practice parameters for the management of colon cancer. Dis Colon Rectum 2012;55:831-843.

5. Labianca R, Nordlinger B, Beretta GD, et al. Early colon cancer: ESMO clinical practice guidelines for diagnosis, treatment and follow-up. Ann Oncol 2013;24(Suppl):64-72.

6. Arbeitsgemeinschaft der Wissenschaftlichen Medizinischen Fachgesellschaften V (AWMF), Deutsche Krebsgesellschaft e.V., Deutsche Krebshilfe e.V. S3-Leitlinie Kolorektales Karzinom, Langversion 1.1, August 2014 AWMF Registrierungsnummer: 021-007OL. Berlin, Germany, 2014. Available at: https://www.awmf.org/leitlinien/detail/l//021-007OL.html. Accessed February 28, 2018

7. Benson AB III, Venook AP, Bekaii-Saab T, et al. NCCN Clinical Practice Guidelines in Oncology: Colon Cancer. Version 2.2016. Accessed August 8, 2016. To view the most recent version, visit NCCN.org.

8. André $T$, Boni $C$, Mounedji-Boudiaf $L$, et al. Oxaliplatin, fluorouracil, and leucovorin as adjuvant treatment for colon cancer. N Engl J Med 2004; 350:2343-2351.

9. Schmiegel W, Pox C, Adler G, et al. S3-Guidelines conference "colorectal carcinoma" 2004 [in German]. Z Gastroenterol 2004;42:1129-1177.

10. Schmoll HJ, Twelves C, Sun W, et al. Effect of adjuvant capecitabine or fluorouracil, with or without oxaliplatin, on survival outcomes in stage III colon cancer and the effect of oxaliplatin on post-relapse survival: a pooled analysis of individual patient data from four randomised controlled trials. Lancet Oncol 2014;15:1481-1492.

11. Hanna NN, Onukwugha E, Choti MA, et al. Comparative analysis of various prognostic nodal factors, adjuvant chemotherapy and survival among stage III colon cancer patients over 65 years: an analysis using Surveillance, Epidemiology and End Results (SEER)-Medicare data. Colorectal Dis 2012;14:48-55.

12. Fata F, Mirza A, Craig G, et al. Efficacy and toxicity of adjuvant chemotherapy in elderly patients with colon carcinoma: a 10-year experience of the Geisinger Medical Center. Cancer 2002;94:1931-1938.

13. Iwashyna TJ, Lamont EB. Effectiveness of adjuvant fluorouracil in clinical practice: a population-based cohort study of elderly patients with stage III colon cancer. J Clin Oncol 2002;20:3992-3998.

14. Zuckerman IH, Rapp T, Onukwugha E, et al. Effect of age on survival benefit of adjuvant chemotherapy in elderly patients with stage III colon cancer. J Am Geriatr Soc 2009;57:1403-1410.

15. Tournigand $\mathrm{C}$, André $\mathrm{T}$, Bonnetain $\mathrm{F}$, et al. Adjuvant therapy with fluorouracil and oxaliplatin in stage II and elderly patients (between ages 70 and 75 years) with colon cancer: subgroup analyses of the Multicenter International Study of Oxaliplatin, Fluorouracil, and Leucovorin in the Adjuvant Treatment of Colon Cancer trial. J Clin Oncol 2012;30: 3353-3360.

16. Hung A, Mullins CD. Relative effectiveness and safety of chemotherapy in elderly and nonelderly patients with stage III colon cancer: a systematic review. Oncologist 2013;18:54-63.

17. Sanoff HK, Carpenter WR, Stürmer T, et al. Effect of adjuvant chemotherapy on survival of patients with stage III colon cancer diagnosed after age 75 years. J Clin Oncol 2012;30:2624-2634.

18. Papamichael D, Audisio RA, Glimelius B, et al. Treatment of colorectal cancer in older patients: International Society of Geriatric Oncology (SIOG) consensus recommendations 2013. Ann Oncol 2015;26:463-476.

19. Haller DG, O'Connell MJ, Cartwright TH, et al. Impact of age and medical comorbidity on adjuvant treatment outcomes for stage III colon cancer: a pooled analysis of individual patient data from four randomized, controlled trials. Ann Oncol 2015;26:715-724.

20. Lemmens VE, van Halteren $\mathrm{AH}$, Janssen-Heijnen $\mathrm{ML}$, et al. Adjuvant treatment for elderly patients with stage III colon cancer in the southern
Netherlands is affected by socioeconomic status, gender, and comorbidity. Ann Oncol 2005;16:767-772.

21. Etzioni DA, El-Khoueiry AB, Beart RW Jr. Rates and predictors of chemotherapy use for stage III colon cancer: a systematic review. Cancer 2008;113:3279-3289.

22. Elferink MA, Wouters MW, Krijnen P, et al. Disparities in quality of care for colon cancer between hospitals in the Netherlands. Eur J Surg Oncol 2010;36(Suppl):S64-73.

23. van Steenbergen LN, Rutten HJ, Creemers GJ, et al. Large age and hospital-dependent variation in administration of adjuvant chemotherapy for stage III colon cancer in southern Netherlands. Ann Oncol 2010;21: 1273-1278.

24. Abraham A, Habermann EB, Rothenberger DA, et al. Adjuvant chemotherapy for stage III colon cancer in the oldest old: results beyond clinical guidelines. Cancer 2013;119:395-403.

25. Kim IY, Kim BR, Kim YW. Factors affecting use and delay ( $\geq 8$ weeks) of adjuvant chemotherapy after colorectal cancer surgery and the impact of chemotherapy-use and delay on oncologic outcomes. PLoS One 2015;10: e0138720

26. Brenner $\mathrm{H}$, Chang-Claude J, Seiler $\mathrm{CM}$, et al. Protection from colorecta cancer after colonoscopy: a population-based, case-control study. Ann Intern Med 2011;154:22-30.

27. Brenner H, Chang-Claude J, Jansen $\mathrm{L}$, et al. Reduced risk of colorectal cancer up to 10 years after screening, surveillance, or diagnostic colonoscopy. Gastroenterology 2014;146:709-717.

28. Weigl K, Jansen L, Chang-Claude J, et al. Family history and the risk of colorectal cancer: the importance of patients' history of colonoscopy. Int J Cancer 2016;139:2213-2220.

29. Jansen L, Hoffmeister M, Chang-Claude J, et al. Age-specific administration of chemotherapy and long-term quality of life in stage II and III colorectal cancer patients: a population-based prospective cohort. Oncologist 2011;16:1741-1751.

30. Hoffmeister $\mathrm{M}$, Jansen $\mathrm{L}$, Rudolph $\mathrm{A}$, et al. Statin use and survival after colorectal cancer: the importance of comprehensive confounder adjustment. J Natl Cancer Inst 2015;107:djv045.

31. Walter V, Jansen L, Hoffmeister M, et al. Smoking and survival of colorectal cancer patients: population-based study from Germany. Int J Cancer 2015;137:1433-1445

32. Walter $\mathrm{V}$, Jansen $\mathrm{L}$, Ulrich $\mathrm{A}$, et al. Alcohol consumption and survival of colorectal cancer patients: a population-based study from Germany. Am J Clin Nutr 2016;103:1497-1506.

33. Daly MC, Hanseman DJ, Abbott DE, et al. Hospital variability in use of adjuvant chemotherapy for patients with stage 2 and 3 colon cancer. Dis Colon Rectum 2016;59:1134-1141.

34. Ko JJ, Kennecke HF, Lim HJ, et al. Reasons for underuse of adjuvant chemotherapy in elderly patients with stage III colon cancer. Clin Colorectal Cancer 2016;15:179-185.

35. Schrag D, Cramer LD, Bach PB, et al. Age and adjuvant chemotherapy use after surgery for stage III colon cancer. J Natl Cancer Inst 2001;93: 850-857.

36. Puts MT, Sattar S, McWatters K, et al. Chemotherapy treatment decisionmaking experiences of older adults with cancer, their family members, oncologists and family physicians: a mixed methods study. Support Care Cancer 2017;25:879-886.

37. Baldwin LM, Dobie SA, Billingsley K, et al. Explaining black-white differences in receipt of recommended colon cancer treatment. J Natl Cancer Inst 2005;97:1211-1220.

38. Wu X, Chen VW, Andrews PA, et al. Treatment patterns for stage III colon cancer and factors related to receipt of postoperative chemotherapy in Louisiana. J La State Med Soc 2004;156:255-261.

39. van Leersum NJ, Janssen-Heijnen ML, Wouters MW, et al. Increasing prevalence of comorbidity in patients with colorectal cancer in the South of the Netherlands 1995-2010. Int J Cancer 2013;132:2157-2163.

40. André T, Boni $C$, Navarro $M$, et al. Improved overall survival with oxaliplatin, fluorouracil, and leucovorin as adjuvant treatment in stage II or III colon cancer in the MOSAIC trial. J Clin Oncol 2009;27:3109-3116. 
Supplemental online content for:

\section{Decreasing Use of Chemotherapy in Older Patients With Stage III Colon Cancer Irrespective of Comorbidities}

Viola Walter, MSc, PhD; Daniel Boakye, MPH; Janick Weberpals, MSc, PhD; Lina Jansen, MSc, PhD; Walter E. Haefeli, MD; Uwe M. Martens, MD; Phillip Knebel, MD; Jenny Chang-Claude, MSc, PhD; Michael Hoffmeister, MSc, PhD; and Hermann Brenner, MD, MPH

J Natl Compr Canc Netw 2019;17(9):1089-1099

eTable 1: Overview of Hospital Characteristics eTable 2: Reasons for Not Administering Adjuvant Chemotherapy According to Physicians eTable 3: Characteristics of Patients Who Refused Chemotherapy eTable 4: Factors Associated With Nonadministration of Adjuvant Chemotherapy 


\section{eTable 1. Overview of Hospital Characteristics}

\begin{tabular}{|c|c|c|c|}
\hline Clinic & Clinic Size, Beds & Surgical Volume ${ }^{a}$ & Certification Status $^{b}$ \\
\hline 1 & $<250$ & $70-149$ & Never certified \\
\hline 2 & $<250$ & $0-69$ & Never certified \\
\hline 3 & $250-749$ & $0-69$ & Never certified \\
\hline 4 & $<250$ & $0-69$ & Never certified \\
\hline 5 & $250-749$ & $70-149$ & Certified before 2017 \\
\hline 6 & 750-999 & $\geq 150$ & Certified before 2017 \\
\hline 7 & $<250$ & $0-69$ & Never certified \\
\hline 8 & $<250$ & $0-69$ & Never certified \\
\hline 9 & $<250$ & $0-69$ & Never certified \\
\hline 10 & 750-999 & $\geq 150$ & Certified before 2017 \\
\hline 11 & $\geq 1,000$ & $\geq 150$ & Certified before 2017 \\
\hline 12 & $250-749$ & 70-149 & Never certified \\
\hline 13 & $<250$ & $0-69$ & Never certified \\
\hline 14 & $<250$ & $70-149$ & Never certified \\
\hline 15 & $250-749$ & $0-69$ & Never certified \\
\hline 16 & $250-749$ & $0-69$ & Never certified \\
\hline 17 & $<250$ & $0-69$ & Never certified \\
\hline 18 & $250-749$ & $70-149$ & Certified before 2017 \\
\hline 19 & $\geq 1,000$ & $\geq 150$ & Never certified \\
\hline
\end{tabular}

aNumber of colon cancer surgeries performed per year.

${ }^{\mathrm{b}}$ Commission on Cancer-certified.
eTable 2. Reasons for Not Administering Adjuvant Chemotherapy According to Physicians

\begin{tabular}{|lcc|}
\hline & $\mathbf{n}(\%)$ & $\begin{array}{c}\text { Mean Age at } \\
\text { Diagnosis (SD), y }\end{array}$ \\
\hline Total & $141(100)$ & $79.2(9.1)$ \\
\hline Patient refusal & $42(29.8)$ & $78.5(8.1)$ \\
\hline Old age & $34(24.1)$ & $85.5(4.1)$ \\
\hline Poor health & $24(17.0)$ & $81.0(8.3)$ \\
\hline Severe secondary diagnosis & $17(12.1)$ & $76.4(10.8)$ \\
\hline Long disease course & $6(4.3)$ & $71.7(14.2)$ \\
\hline Death & $4(2.8)$ & $79.0(14.9)$ \\
\hline Wound healing disorder & $2(1.4)$ & $67.0(2.8)$ \\
\hline Only follow-up care recommended & $1(0.7)$ & $86.0(0)$ \\
\hline Postoperative sepsis & $1(0.7)$ & $69.0(0)$ \\
\hline No reason given & $39(27.7)$ & $77.3(9.4)$ \\
\hline Several of the above & $25(17.7)$ & $81.1(8.2)$ \\
\hline
\end{tabular}




\section{eTable 3. Characteristics of Patients Who Refused} Chemotherapy ( $N=42)$

\begin{tabular}{|lccc|}
\hline Variable & $\begin{array}{c}\text { Chemotherapy } \\
\text { Nonrecipients, } \mathbf{n}\end{array}$ & $\begin{array}{c}\text { Refused } \\
\text { Chemotherapy, } \mathbf{n}\end{array}$ & Row \% \\
\hline \begin{tabular}{l} 
Sex \\
\hline Female
\end{tabular} & 64 & 20 & 31.3 \\
\hline Male & 77 & 22 & 28.6 \\
\hline Age, y & & & \\
\hline $\begin{array}{l}30-64 \\
65-74\end{array}$ & 11 & 3 & 27.3 \\
\hline$\geq 75$ & 22 & 7 & 31.8 \\
\hline
\end{tabular}

Number of comorbidities ${ }^{\mathrm{a}}$

\begin{tabular}{lrrr}
\hline 0 & 32 & 8 & 25.0 \\
\hline 1 & 46 & 14 & 20.4 \\
\hline 2 & 63 & 20 & 31.8
\end{tabular}

Period of diagnosis

\begin{tabular}{rrrr}
\hline $2003-2004$ & 27 & 6 & 22.2 \\
\hline $2005-2008$ & 44 & 15 & 34.1 \\
\hline $2009-2012$ & 70 & 21 & 30.0
\end{tabular}

Surgical volume

\begin{tabular}{llcl}
\hline Low & 45 & 14 & 31.1 \\
\hline Medium & 34 & 12 & 35.3 \\
\hline High & 62 & 16 & 25.8
\end{tabular}

CoC-certified center status (at diagnosis/in 2017)

\begin{tabular}{lrrr}
\hline No/No & 71 & 23 & 32.3 \\
\hline No/Yes & 26 & 5 & 19.2 \\
\hline Yes/Yes & 14 & 14 & 31.8 \\
\hline
\end{tabular}

Abbreviation: CoC, Commission on Cancer.

aalncluding diabetes, hypertension, myocardial infarction, angina pectoris, heart failure, stroke, and cerebrovascular disorders. 


\section{eTable 4. Factors Associated With Nonadministration of Adjuvant Chemotherapy ${ }^{\mathrm{a}}$}

No Chemotherapy vs Chemotherapy

\begin{tabular}{|c|c|c|c|c|}
\hline & \multirow[b]{2}{*}{ Patients, n } & \multirow[b]{2}{*}{ Events, $\mathbf{n}$} & \\
\hline & & & OR $^{b}(95 \% \mathrm{CI})$ & ORc $(95 \% \mathrm{Cl})$ \\
\hline \multicolumn{5}{|l|}{ Sex } \\
\hline Female & 278 & 44 & Ref & Ref \\
\hline Male & 309 & 55 & $1.34(0.81-2.22)$ & $1.95(0.97-3.93)$ \\
\hline \multicolumn{5}{|l|}{ Age at diagnosis, y } \\
\hline $30-64$ & 190 & 8 & Ref & Ref \\
\hline $65-74$ & 208 & 15 & $1.73(0.71-4.24)$ & $1.54(0.59-4.05)$ \\
\hline$\geq 75$ & 189 & 76 & 15.5 (7.12-33.9) & $12.5(5.19-30.3)$ \\
\hline \multicolumn{5}{|l|}{$\mathrm{BMI}, \mathrm{kg} / \mathrm{m}^{2}$} \\
\hline$<25.0$ & 235 & 46 & Ref & Ref \\
\hline $25.0-29.9$ & 241 & 42 & $0.82(0.48-1.41)$ & $0.82(0.45-1.49)$ \\
\hline$\geq 30.0$ & 111 & 11 & $0.54(0.25-1.18)$ & $0.48(0.21-1.11)$ \\
\hline \multicolumn{5}{|c|}{ Number of comorbidities ${ }^{d}$} \\
\hline 0 & 225 & 24 & Ref & Ref \\
\hline 1 & 203 & 32 & $1.17(0.61-2.25)$ & $1.12(0.56-2.22)$ \\
\hline$\geq 2$ & 159 & 43 & $2.17(1.13-4.17)$ & $2.09(1.03-4.21)$ \\
\hline \multicolumn{5}{|l|}{ Smoking status } \\
\hline Never-smoker & 309 & 51 & Ref & Ref \\
\hline Former smoker & 215 & 42 & $1.59(0.94-2.69)$ & $1.55(0.82-2.92)$ \\
\hline Current smoker & 63 & 6 & $1.35(0.48-3.78)$ & $1.25(0.42-3.73)$ \\
\hline
\end{tabular}

Education levele

\begin{tabular}{llll}
\hline Low $(<9 \mathrm{y})$ & 127 & 33 & Ref \\
\hline Medium $(9-10 y)$ & 332 & 51 & $0.82(0.46-1.47)$ \\
\hline High $(>10 y)$ & 128 & 15 & $0.71(0.37-1.39)$ \\
\hline
\end{tabular}

Living with a partner

\begin{tabular}{|c|c|c|c|c|}
\hline Yes & 444 & 59 & Ref & Ref \\
\hline No & 143 & 40 & $1.61(0.94-2.75)$ & $1.96(1.04-3.69)$ \\
\hline \multicolumn{5}{|c|}{ Period of diagnosis } \\
\hline 2003-2004 & 136 & 21 & Ref & Ref \\
\hline 2005-2008 & 235 & 29 & $0.75(0.38-1.50)$ & $0.62(0.30-1.29)$ \\
\hline 2009-2012 & 216 & 49 & $1.82(0.94-3.50)$ & $1.67(0.83-3.37)$ \\
\hline
\end{tabular}

Surgical volume

\begin{tabular}{|c|c|c|c|c|}
\hline Low & 140 & 31 & Ref & Ref \\
\hline Medium & 185 & 22 & $0.52(0.27-1.00)$ & $0.40(0.19-0.83)$ \\
\hline High & 262 & 46 & $0.73(0.41-1.30)$ & $0.65(0.35-1.20)$ \\
\hline \multicolumn{5}{|c|}{ CoC-certified center status (at diagnosis/in 2017) ${ }^{f}$} \\
\hline $\mathrm{No} / \mathrm{No}$ & 277 & 48 & Ref & Ref \\
\hline No/Yes & 173 & 21 & $0.61(0.33-1.12)$ & $0.70(0.36-1.34)$ \\
\hline Yes/Yes & 137 & 30 & $1.13(0.64-2.00)$ & $0.83(0.41-1.66)$ \\
\hline
\end{tabular}

Abbreviations: BMI, body mass index; CoC, Commission on Cancer; OR, odds ratio.

aExcluding patients who refused chemotherapy $(n=42)$.

${ }^{b}$ Adjusted for age and hospitals where patients were treated.

cAdditional adjustment for sex, BMI, T stage, N stage, number of comorbid conditions, smoking status, education level, living with a partner, and period of diagnosis.

'Including diabetes, hypertension, myocardial infarction, angina pectoris, heart failure, stroke, and cerebrovascular disorders.

eYears of school education.

${ }^{f}$ Hospital where patients were treated was removed from the model. 